\section{AB1359-HPR THE COMBINED EFFECTS OF LIFESTYLE HABITS ON HEALTH-RELATED QUALITY OF LIFE, PHYSICAL AND MENTAL FUNCTIONS IN PATIENTS WITH SPONDYLOARTHRITIS}

Elisabeth Mogard ${ }^{12}$, Ann Bremander ${ }^{1} 3{ }^{4}$, Emma Haglund ${ }^{2}$. . ${ }^{1}$ Lund University, Department of Clinical Sciences Lund, Rheumatology, Lund, Sweden; ${ }^{2} R \& D$ Spenshult, Halmstad, Sweden; ${ }^{3}$ Department of Regional Health Research, University of Southern Denmark, Odense, Denmark; ${ }^{4}$ Danish Hospital for Rheumatic Diseases, University Hospital of Southern Denmark, Sønderborg, Denmark; ${ }^{5} \mathrm{Halmstad}$ University, School of Business, Engineering and Science, Halmstad, Sweden

Background Earlier studies have found strong correlations between worse health and an unhealthy lifestyle, such as not meeting recommendations of moderate-to-vigorous physical activity, being overweight or obese and the use of tobacco in patients with spondyloarthritis $(\mathrm{SpA})$. The impact of more than one unhealthy lifestyle habit (LsH) is however, scarcely described.

Objectives To study the combined effects of unhealthy LsHs on health-related quality of life (HRQoL) and physical and mental functions in patients with SpA. Differences between SpA subgroups and gender were also studied.

Methods Postal questionnaires were in 2009 and 2011 sent to all patients diagnosed with $\mathrm{SpA}$ and registered in the Skane Healthcare Register. This study included patients who at both time points responded to the survey, were $\geq 20$ years, and had ankylosing spondylitits (AS), psoriatic arthritis (PsA) or undifferentiated spondyloarthritis (USpA). Cross-sectional data from the 2011 questionnaire were available for 1601 patients (AS $n=455$, PsA $n=883$, USpA $n=263$ ), with a mean age of 58 (13) years ( $52 \%$ women). Self-reported levels of weekly physical activity at moderate or vigorous intensity, (MVPA), use of tobacco (cigarettes and/ or snuff) and BMI (overweight or obese) were dichotomized as "healthy" or "unhealthy". The number of unhealthy LsH were then summarized and stratified into four groups (scoring $0-3,0=$ no unhealthy LsH). HRQoL was assessed with EQ-5D (0-1, worst-best), and physical function with BASFI. Disease activity (BASDAI), pain, fatigue (0-10, best-worst), anxiety, and depression (HADa/d) $(0-21$, no distress-maximum distress) were also measured. Statistical analyses were performed with Chi Square test and ANOVA.
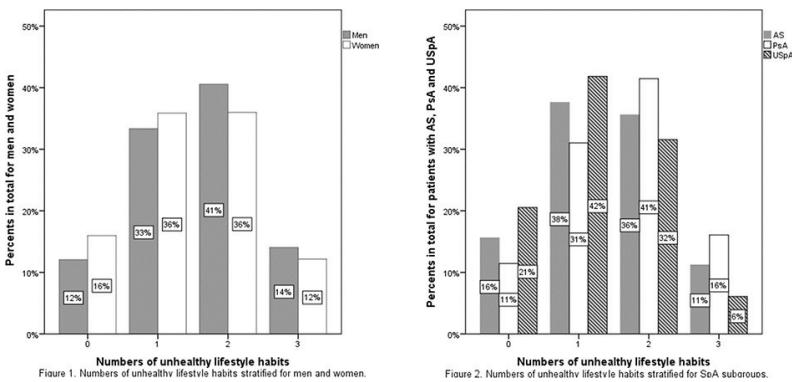

Results Fourteen percent $(n=226)$ reported none of the studied unhealthy LsH, while $35 \%(n=555)$ reported one, $38 \%(n=611)$ two, and $13 \%(n=209)$ three unhealthy LsH. Reports of one and more unhealthy LsH had increasing negative impact on HRQoL (from mean 0.74 (SD 0.19 ) to $0.57(0.30)$ ), disease activity (from $3.2(2.1)$ to $4.5(2.3)$ ), physical function $(2.3(2.1)$ to $4.4(2.6))$, VAS-pain (3.4 (2.3) to $4.8(2.5))$, VAS-fatigue (4.2 (2.7) to $5.5(2.7))$, anxiety (4.8 (4.2) to $5.6(4.4)$ ) and depression (3.3 (3.3) to $4.8(3.8))$ in patients with $\mathrm{SpA}(\mathrm{p}=0.019$-Conclusion Our findings support that the combined effect of unhealthy lifestyle habits have negative impact on many aspects of health. There is a need for interventions aiming at screening for not only one but several unhealthy lifestyle habits combined, and to offer coaching to increase behavioral change and promote better health. Disclosure of Interests None declared

DOI: 10.1136/annrheumdis-2019-eular.1985

\section{AB1360-HPR PREVALENCE OF TOBACCO AND ALCOHOL COMSUMPTION IN PATIENTS WITH RHEUMATOID ARTHRITIS}

Laura Villarreal ${ }^{1}$, Michael Cabrera ${ }^{2}$, Pedro Santos-Moreno ${ }^{3}$, Diana BuitragoGarcia ${ }^{4}{ }^{1}$ Biomab - Center for rheumatoid arthritis, Psychology, Bogotá, Colombia; ${ }^{2}$ Biomab - Center for rheumatoid arthritis, EHR administration, Bogotá, Colombia; ${ }^{3}$ Biomab - Center for rheumatoid arthritis, Rheumatology, Bogotá, Colombia; ${ }^{4}$ Biomab - Center for rheumatoid arthritis, Nursing research, Bogotá, Colombia

Background: Rheumatoid arthritis (RA) is a chronic, inflammatory arthritis leading to progressive joint and organ system damage and disability. It has been demonstrated that smoking and alcohol consumption is a risk factor for poor response to RA treatment.
Objectives: To describe the prevalence of smoking and alcohol consumption in patients with rheumatoid arthritis.

Methods: We conducted a retrospective study, patients were followed during 12 months. Smoking and alcohol consumption were assessed through yearly questionnaires. The disease activity and functional status were measured annually by the DAS28. We calculated means, and standard deviations for continuous variables and categorical variables were presented as rates. We estimated the prevalence smoking and alcohol consumption and explored if there was an association between DAS28 and smoking or alcohol consumption.

Results: We included 6491 patients. patients, $82 \%$ were female and $18 \%$ male; median age was 60 years RIQ (50-67), regarding disease activity, mean DAS28 was $2.59 \pm 1.08$. The prevalence of alcohol consumptions was $2.26 \%$ while smoking $7.83 \%$. We did no find an statistical association between smoking alcohol consumption and DAS28.

Conclusion: Our study showed a small prevalence of patients with rheumatoid arthritis who smoke or consume alcohol without any associations between smoking or alcohol consumption with disease activity. This could be attributed to a low prevalence in our study. Further research is needed in order to propose other methodological approaches to explore this association.

Disclosure of Interests:

Laura Villarreal: None declared, Michael Cabrera: None declared, Pedro Santos-Moreno Grant/research support from: $\mathrm{Dr}$ Santos has received research grants from Janssen, Abbvie and UCB, Speakers bureau: $\mathrm{Dr}$ Santos has received speaker fees from Sanofi, Lilly, Bristol, Pfizer, Abbvie, Janssen and UCB, Diana Buitrago-Garcia: None declared

DOI: 10.1136/annrheumdis-2019-eular.7360

\section{HPR Interventions (educational, physical, social and psychological)}

\section{AB1361-HPR BENEFITS OF HYDROGINASTICA IN ELDERLY WITH OSTEOPOROSIS}

Mateus Antunes ${ }^{1}$, Sonia Bertolini ${ }^{2}$, Amelia Pasqual Marques ${ }^{1} .{ }^{1}$ University of São Paulo, Department of Physical Therapy, Speech Therapy and Occupational Therapy, São Paulo, Brazil, ${ }^{2}$ University Center of Maringá - UNICESUMAR, Department of Health Promotion, Maringá - PR, Brazil

Background: The aging of the population has repercussions in several social sectors, among them health, with a view to increasing the frequency of Chronic Non-Communicable Diseases. It is worth highlighting Osteoporosis, since it is associated with increased mortality and dependencies, becoming a growing public health problem. As a form of maintenance and prevention of osteoporosis it is indicated the performance of physical exercises, among them, the water gymnastics stands out.

Objectives: The study aimed to present and discuss studies on the benefits of water aerobics for the elderly with osteoporosis.

Methods: For the development of the present study a bibliographic research was carried out in the years 2010 to 2017 through searches in national and international journals indexed in PubMed, Scielo, Lilacs and Medline databases. The descriptors used were: Elderly, Aging Process, Hydrogeology, Aquatic Activity, Osteoporosis and Health Promotion in Portuguese, Spanish and English. The search period was between the months of February and March of 2017 and after selecting the articles, to extract the definitions about the theme, they were read.

Results: We can identify that water aerobics is a practice that addresses these benefits in the elderly with osteoporosis. Another aspect found in the studies is that water gymnastics provides the elderly with improved socio-affective relationships.

Even considering the natural losses due to aging on some physical characteristics, water aerobics is an important exercise practice option. The activities in the aquatic environment are indicated for a considerable gain of muscular mass and reduction of the loss of the same one, aiding in the tonification of the musculature, the loss of fat and the increase of the resistance. For the elderly with osteoporosis this modality is a preventive aid that contributes to improve their quality of life.

Among the studies found, one performed in Japan in the elderly with osteoporosis, found an increase in VO2max, flexibility and increased strength of hydrogymnastics participants; in Israel, the elderly women reduced the Muscle Mass Index and body fat. In Brazil, a study verified the improvement of functional capacity in elderly women practicing water aerobics. 
Water aerobics for the elderly has been gaining momentum and growing numbers of fans, so much so that in the last 10 years the popularity of exercise in the aquatic environment has increased significantly.

Conclusion: With the present study it can be verified that the hydroginastic presents numerous benefits for maintenance and prevention of osteoporosis in the elderly. Regular practice of water aerobics contributes to good body health, as well as providing physical and mental well-being and social interaction among its practitioners. This study may provide important health professionals with knowledge about the importance of encouraging and intensifying regular physical exercise, including hydrogymnastics, as one of the determinants of elderly health promotion.

\section{REFERENCES}

[1] Teixeira CS, Lemos FFC, Lopes LFD, Rossi AG, Mota CB. Corporal balance and physical exercises: an investigation in elderly women who practice different exercise modalities. Acta Fisiátrica. 2008;15(3):156-159.

[2] Kemper, C., Oliveira, R. J. D., Bottaro, M., Moreno, R., Bezerra, L. M. A., Guido, M., \& França, N. M. D. Effects of swimming and resistance training on bone mineral density of older women. Revista Brasileira de Medicina do Esporte. 2009;15(1):10-13.

Acknowledgement: Instituto Cesumar de Ciência, Tecnologia e Inovação (ICETI)

Disclosure of Interests: None declared

DOI: 10.1136/annrheumdis-2019-eular.2463

\section{AB1362-HPR THE EFFECTS OF 4 - WEEK REHABILITATION PROGRAM ON PATIENT OUTCOMES IN PATIENTS WITH KNEE OSTEOARTHRITIS}

Onur Aydogdu, Sefer Kılıçkap, Beyzanur Daşkın, Zübeyir Sarı. Marmara University, Faculty of Health Sciences, Department of Physiotherapy and Rehabilitation, Istanbul, Turkey

Background: Osteoarthritis (OA) is the most common form of arthritis and a major cause of pain and disability in older adults (1). Older adults with knee OA report lower physical functioning and increased difficulty in performing activities of daily living compared with older adults without knee OA (2). Knee OA treatment aims to improve physical functions, quality of life, prevent disability, and decrease pain. Therapeutic exercises and physiotherapy treatment applications are recommended for the patients with knee OA (3)

Objectives: The purpose of this study was to examine the effects of 4 week physiotherapy and rehabilitation program on range of motion, balance, knee swelling, and activities of daily life, quality of life, and functional status in patients with knee osteoarthritis. It was hypothesized that 4 - week rehabilitation program might provide improvements in measurements of the patient outcomes in patients with knee osteoarthritis.

Methods: Twenty-seven healthy subjects were participated in the study. Subjects had no surgery history of lower extremity. Range of motion, balance, knee swelling, activities of daily life, quality of life, and functional status were assessed by Goniometer, Berg Balance Test, Tape measure, Barthel Index, WHOQL Scale, WOMAC Scale, respectively. The physiotherapy and rehabilitation program started after the first assessment and was applied as 20 sessions for a total of four weeks, five sessions per week. All of the assessments procedures were performed again after the treatment.

Results: There were statistically significant improvements in measures of range of motion, balance, knee swelling, and functional status between pre- and post - treatment $(p<0.05)$. However, no significant difference was found in activities of daily life and quality of life after the 4-week rehabilitation program ( $p>0.05$ ).

Conclusion: Based on our findings, short-term effects of physiotherapy and rehabilitation program may be beneficial on range of motion, balance, knee swelling, and functional status in patients with knee osteoarthritis. However, 4-week rehabilitation program has no effect on activities of daily life and quality of life in patients with knee osteoarthritis.

\section{REFERENCES}

[1] Huang L, Guo B, Xu F, Zhao J. Effects of quadriceps functional exercise with isometric contraction in the treatment of knee osteoarthritis. Int $\mathrm{J}$ Rheum Dis. 2018 May;21(5):952-959.

[2] Mihalko SL, Cox P, Beavers DP, et al. Effect of intensive diet and exercise on self-efficacy in overweight and obese adults with knee osteoarthritis: The IDEA randomized clinical trial. Transl Behav Med. 2018 Apr 4.
[3] Aydoğdu O, Sari Z, Yurdalan SU, Polat MG. Clinical outcomes of kinesio taping applied in patients with knee osteoarthritis: A randomized controlled trial. J Back Musculoskelet Rehabil. 2017 Sep 22;30(5):1045-1051.

Disclosure of Interests: None declared

DOI: 10.1136/annrheumdis-2019-eular.7500

\section{AB1363-HPR THE INVESTIGATION OF THE QUALITY OF LIFE AND FUNCTIONAL ABILITIES IN PATIENTS WITH JUVENILE SCLERODERMA}

Arzu Dag ${ }^{1}$, Ela Tarakci ${ }^{2}$, Amra Adrovic ${ }^{3}$, Ozgur Kasapcopur ${ }^{4} .{ }^{1}$ Istanbul Yeni Yuzyil University, Faculty of Health Science, Department of Physiotherapy and Rehabilitation, Istanbul Turkey, Istanbul, Turkey; ${ }^{2}$ Istanbul University-Cerrahpasa, Faculty of Health Science, Department of Physiotherapy and Rehabilitation, Department of Neurologic Physiotherapy and Rehabilitation, Istanbul, Turkey, ${ }^{3}$ Istanbul University-Cerrahpasa, Cerrahpasa Medical School, Department of Pediatric Rheumatology, Istanbul, Turkey, ${ }^{4}$ Istanbul University-Cerrahpasa, Cerrahpasa Medical School, Department of Pediatric Rheumatology, Istanbul, Turkey

Background: Juvenile scleroderma (JS) is a rarely seen chronic connective tissue disorder. There are two main disease forms: juvenile localized scleroderma (JLS) and juvenile systemic sclerosis (JSS). These conditions share common pathophysiologic features which are mainly characterized by inflammation and fibrosis of the skin. In JLS, fibrosis involves restricted areas of the skin, whereas in JSS it also affects the interna organs

Objectives: There have been few studies of quality of life in pediatric scleroderma and these focused predominantly on self-perception and the influence of skin involvement. Our cross-sectional study aimed to describe the influence of juvenile scleroderma on functional ability and quality of life in relation to clinical and demographic measures.

Methods: 30 patients ( 26 girls, 4 boys) with JS between the ages of 618 years and 30 healthy controls ( 20 girls, 10 boys) with similar age and gender were included in our study. Patients with either localized scleroderma or systemic sclerosis (SSc) attending pediatric rheumatology clinics, together with their parents were asked to complete a set of 3 validated measures. Children completed their functional abilitiy status with Childhood Health Assessment Questionnaire (CHAQ) and Jebson Taylor Hand Function Test (JTHFT). The quality of life were evaluated with Scleroderma Health Assessment Questionnaire (SHAQ). Clinical and demographic data were provided by consultant pediatric rheumatologists and physiotherapists.

Results: The mean age of the JS group was $14.06 \pm 3.24$ years $(86 \%$ female, $14 \%$ male; while the $\% 53$ localized scleroderma, $\% 47 \mathrm{SSc}$ ) and of the control group was $12.43 \pm 3.24$ years. There were significantly differences between the two groups in functional ability scores (JTHFT) (CHAQ) and quality of life scores (SHAQ) $(p<0,05)$. In JS grup, the total$\mathrm{CHAQ}$ score was $0.47 \pm 0,63$ (range $0-3,0$ indicating no impairment), the JTHFT-Total left hand and right hand scores were 44,05 $\pm 9,83$ and 41,49 $\pm 7,42$ second respectively, and the median SHAQ-Total score was 0,34 (range $0-6,0$ indicating no impairment).

Conclusion: Functional ability disabilities in JS patients cause limitations in daily living activity. Scleroderma had only a moderate effect on quality of life and functional abilites as measured by the 3 validated instruments. Although a small number of children reported greater impairment, this is an encouraging finding, given its potential disfiguring and debilitating effects.

\section{REFERENCES}

[1] Lam C, Young N, Marwaha J, McLimont M, Feldman BM. Revised versions of the Childhood Health Assessment Questionnaire (CHAQ) are more sensitive and suffer less from a ceiling effect. Arthritis Care Res. $2004 ; 51(6): 881-9$.

[2] Reedman SE, Beagley S, Sakzewski L, Boyd RN. The Jebsen Taylor Test of Hand Function: A Pilot Test-Retest Reliability Study in Typically Developing Children. Phys Occup Ther Pediatr. 2016; 36(3):292-304.

[3] Rocha, L. F., Marangoni, R. G., Sampaio-Barros, P. D., Levy-Neto, M. Yoshinari, N. H., Bonfa, E.,. .. \& Kowalski, S. C. Cross-cultural adaptation and validation of the Brazilian version of the Scleroderma Health Assessment Questionnaire (SHAQ). Clinical rheumatology. 2014; 33(5):699-706.

*The co-author Ela Tarakcl was supported to participate to EULAR 2019

by Turkish Academy of Sciences as a winner of the Young Scientists

Award Programme (GEBIP) 2018.

Disclosure of Interests: None declared

DOI: 10.1136/annrheumdis-2019-eular.7412 